\title{
Lúpus Eritematoso Sistêmico Senil: relato de caso e revisão da literatura
}

\author{
Senile Systemic Lupus Erythematosus: case report and literature review \\ Lupus eritematoso sistémico senil: reporte de un caso y revisión de la literatura
}

Recebido: 25/08/2021 | Revisado: 30/09/2021 | Aceito: 09/09/2021 | Publicado: 11/09/2021

Camila Aragão Borges

ORCID: https://orcid.org/0000-0001-9850-3412

Hospital Geral de Fortaleza, Brasil

E-mail: camilaaragaob@gmail.com

Larissa Oliveira Ribeiro

ORCID: https://orcid.org/0000-0003-0557-9851

Centro Universitário Christus, Brasil

E-mail:larissaolivs0103@gmail.com

Marla Rochana Braga Monteiro

ORCID: https://orcid.org/0000-0001-8602-3999 Universidade Estadual do Ceará, Brasil E-mail: m.marlarochana@gmail.com

Fabrício André Martins da Costa

ORCID: https://orcid.org/0000-0002-7080-1386 Hospital Geral Doutor Waldemar de Alcântara, Brasil E-mail: fabricioamc18@gmail.com

Ítalo Crizóstomo Lima

ORCID: https://orcid.org/0000-0002-2298-8940 Hospital Geral Doutor Waldemar de Alcântara, Brasil E-mail: italoicl@hotmail.com

Kristopherson Lustosa Augusto ORCID: https://orcid.org/0000-0001-9254-9129 Universidade Federal do Ceará, Brasil E-mail: kristopherson@hotmail.com

\section{Resumo}

Introdução: O início do Lúpus Eritematoso Sistêmico (LES), após 50 anos de idade, ocorre em 3 a $18 \%$ dos pacientes e é denominado Lúpus Tardio ou Lúpus Senil (LESS). Objetivo: descrever o caso clínico de um paciente diagnosticado com Lúpus Eritematoso Sistêmico Senil que esteve internado no Hospital Geral Doutor Waldemar de Alcântara, além de fazer uma revisão bibliográfica sobre o tema a fim de compartilhar com a comunidade médica a experiência e o conhecimento obtidos. Métodos: Estudo descritivo do tipo relato de caso, utilizando-se informações obtidas através de interação com o paciente durante a internação, bem como dados obtidos no prontuário dos resultados de exames laboratoriais e de imagens. Discussão: A gravidade do lúpus diminui com a idade. Alguns estudos evidenciaram que pessoas acometidos por LES de início tardio apresentam menor envolvimento de órgãos específicos em comparação aos pacientes de início precoce. Da mesma forma, eles apresentam mais frequentemente serosite, síndrome de Sjögren e manifestações neurológicas. Conclusão: Apesar de rara, a hipótese diagnóstica de LESS deve ser lembrada como diagnóstico diferencial. Para isso, é necessária a solicitação precoce do FAN (fator antinuclear), que é critério mandatório do lúpus. Dado o diagnóstico precoce do LESS, o paciente pode beneficiarse com um menor tempo de internação, além de diminuir o uso de medicamentos desnecessários.

Palavras-chave: Lúpus Eritematoso Sistêmico; Diagnóstico tardio; Anticorpos antinucleares; Gravidade do paciente.

\begin{abstract}
Introduction: The onset of Systemic Lupus Erythematosus (SLE), after 50 years of age, occurs in 3 to $18 \%$ of patients and is called Lupus Late or Lupus Senile (LESS). Objective: to describe the clinical case of a patient diagnosed with Senile Systemic Lupus Erythematosus who was admitted to the Doctor Waldemar de Alcântara General Hospital, in addition to conducting a literature review on the subject in order to share the experience and knowledge obtained with the medical community. Methods: Descriptive study of the case report type, using information obtained through interaction with the patient during hospitalization, as well as data obtained from the medical record of the results of laboratory tests and images. Discussion: The severity of lupus decreases with age. Some studies have shown that patients with late-onset SLE have less specific organ involvement compared to early-onset patients. Likewise, they more often present with serositis, Sjögren's syndrome and neurological manifestations. Conclusion: Although rare, the diagnostic hypothesis of LESS should be considered as a differential diagnosis. For this, it is necessary to request na early FAN (antinuclear factor), which is a mandatory criterion for lupus. Given the early diagnosis of LESS, the patient can benefit from a shorter hospital stay, in addition to avoiding the use of unnecessary medications.

Keywords: Lupus Eritematoso Systemic; Delayed Diagnosis; Antibodies antinuclear; Patient acuity.
\end{abstract}




\section{Resumen}

Introducción: La aparición del Lupus Eritematoso Sistémico (LES), después de los 50 años, ocurre en el 3 al $18 \%$ de los pacientes y se denomina Lupus Tardío o Lupus Senil (LESS). Objetivo: describir el caso clínico de un paciente diagnosticado de Lupus Eritematoso Sistémico Senil que ingresó en el Hospital General Doctor Waldemar de Alcântara, además de realizar una revisión de la literatura sobre el tema con el fin de compartir la experiencia conocimientos obtenidos con el médico. comunidad. Métodos: Estudio descriptivo del tipo reporte de caso, utilizando información obtenida a través de la interacción con el paciente durante la hospitalización, así como datos obtenidos de la historia clínica de los resultados de las pruebas de laboratorio e imágenes. Discusión: La gravedad del lúpus disminuye con la edad. Algunos estudios han demostrado que los pacientes con LES de inicio tardío tienen una afectación orgánica menos específica en comparación con los pacientes de inicio temprano. Asimismo, presentan con mayor frecuencia serositis, síndrome de Sjögren y manifestaciones neurológicas. Conclusión: Aunque es poco común, la hipótesis diagnóstica de LESS debe considerarse como un diagnóstico diferencial. Para ello, es necesario solicitarun FAN (factor antinuclear) temprano, que es un criterio obligatorio para el lupus. Dado el diagnóstico precoz de LESS, el paciente puede beneficiarse de una estancia hospitalaria más corta, además de evitar el uso de medicamentos innecesarios.

Palabras clave: Lupus Eritematoso Sistémico; Diagnóstico tardío; Lupus anticoagulante; Gravedad del paciente.

\section{Introdução}

O Lúpus Eritematoso Sistêmico (LES) é uma doença autoimune que acomete múltiplos órgãos, com inúmeras manifestações imunológicas e clínicas, envolvendo pele, articulações, rins, células sanguíneas e sistema nervoso. O LES é frequentemente descrito como uma doença que atinge com mais frequência pacientes do sexo feminino em idade reprodutiva, com distribuição étnica universal, apesar de parecer haver uma predileção por afrodescendentes (Arnaud et al, 2012). Na maioria das vezes a doença é herdada de maneira poligênica a partir de interações genéticas com fatores ambientais, como exposição à luz ultravioleta, infecção pelo vírus Epstein-Barr e fatores hormonais, resultando em desregulação imunológica ao nível de citocinas, células T, células B e macrófagos. (Kaul, A.; Gordon, C., 2016)

O início do LES, após a idade de 50 anos, ocorre em 3 a 18\% dos indivíduos acometidos pela doença e é denominado Lúpus Tardio ou Lúpus Senil (LESS), estando associado à etnia caucasiana e ao sexo feminino. O LESS afeta principalmente mulheres, tal diferença é mais expressiva entre mulheres e homens jovens do que entre mulheres e homens mais velhos (10: $1-$ 4: 1), com uma média de 5: 1. Essa redução da proporção está associada à mudança nos valores de estrogênio, com pico na idade reprodutiva. As mulheres menopausadas e os homens senis que desenvolvem LESS apresentam início da doença mais insidioso, com manifestações clínicas incomuns e redução da atividade da doença (Estel, GJP, 2017; Riveros, 2020).

O diagnóstico de LESS é, muitas vezes, tardio, sendo estabelecido apenas no curso de investigações mais extensas. Apesar desse aparente curso benigno, estudos recentes abordaram o impacto negativo que a idade mais avançada, no início da doença, pode ter sobre o resultado do lúpus em termos de morbimortalidade, possivelmente, devido a circunstâncias relacionadas à idade, como maior número de comorbidades e maior exposição aos riscos cardiovasculares.

As opções atuais de tratamento para o LES são antimaláricos, anti-inflamatórios esteroidais e não esteroidais, drogas imunossupressoras e imunobiológicos. O manejo da patologia em pacientes mais velhos depende do tipo e da gravidade das manifestações da doença (Lalani, 2010).

O reconhecimento adequado e a distinção do LES de início precoce são importantes, pois o manejo adequado pode melhorar o prognóstico nesses pacientes idosos.

O presente estudo tem por objetivo descrever o caso clínico de um paciente diagnosticado com Lúpus Eritematoso Sistêmico Senil que esteve internado no Hospital Geral Doutor Waldemar de Alcântara. 


\section{Metodologia}

\section{Desenho do Estudo}

Trata-se de um estudo descritivo do tipo relato de caso, utilizando-se informações obtidas, através de interação com o paciente, durante a internação, bem como dados obtidos no prontuário dos resultados de exames laboratoriais e de imagens. Para realizar um estudo de caso, foi feito assim como Pereira A. S. et al.(2018). a análise do caso, analisando qual seria o fenômeno relevante que apresentasse interesse para algum grupo ou para a sociedade. Foi preciso então identificar, que características e/ou importância tornam o estudo um caso. Essa identificação inclui a definição de um problema a ser estudado.

\section{Local do estudo}

O Hospital Geral Dr. Waldemar Alcântara (HGWA) é um hospital de atenção secundária, o único da rede pública em Fortaleza a receber o título de Acreditado com Excelência por nível III, pela Organização Nacional de Acreditação (ONA). Oferece apoio para a rede terciária de assistência do Estado do Ceará, atendendo exclusivamente pacientes do Sistema Único de Saúde (SUS). Possui 336 leitos, abrangendo as áreas da clínica médica, cirurgia e pediatria. Conta também com Unidades de Terapia Intensiva adulto, neonatal e pediátrica, berçário de médio risco e duas Unidades de Cuidados Especiais.

O HGWA desenvolve ainda programas de assistência ambulatorial e domiciliar, assim como cuidados especiais e personalizados, com equipes multidisciplinares para pacientes portadores de enfermidades crônicas.

\section{População alvo}

Um único indivíduo, do sexo masculino, com diagnóstico de Lúpus Eritematoso Sistêmico Senil durante internação no Hospital Geral Dr. Waldemar Alcântara (HGWA), de janeiro a fevereiro de 2020.

\section{Técnica e instrumento de coleta de dados}

Revisão do prontuário para descrição do caso clínico e revisão de literatura utilizando as bases científicas do PubMed/MEDLINE.

\section{Aspectos Éticos}

A pesquisa será submetida ao Comitê de Ética da Escola de Saúde Pública de Fortaleza e estará respeitando os princípios éticos de pesquisa envolvendo seres humanos da Resolução 466/12 do Conselho Nacional de Saúde (BRASIL, 1996).

\section{Caso Clínico}

Paciente do sexo masculino, 68 anos, natural e procedente de Pacoti - Ceará, solteiro, católico, pardo, ex-agricultor. Previamente hipertenso e dislipidêmico, fazia uso domiciliar de captopril e sinvastatina. Admitido no Hospital Instituto Dr. José Frota (IJF) no dia 31/10/2019 com síndrome edemigênica a esclarecer, evoluindo durante o internamento com diversas intercorrências infecciosas, como infecção do trato urinário e infecção de sítio de cateter de hemodiálise, com prescrição de antibioticoterapia, incluindo Meropenem e Daptomicina.

Durante o internamento no IJF, apresentou também proteinúria (3+), hipoalbuminemia (albumina: $2 \mathrm{~g} / \mathrm{dL}$ ) e disfunção renal (aumento de escórias nitrogenadas), com necessidade de realização de terapia de substituição renal. Após algumas sessões de hemodiálise, o paciente evoluiu com melhora parcial do edema e da dispneia. Além disso, obteve melhora significativa das escórias nitrogenadas, conseguindo suspensão da diálise. 
Apresentava também derrame pleural bilateral e derrame pericárdico volumoso, com diminuição do enchimento ventricular, sem melhora após restrição hídrica e terapia de substituição renal. Por isso, realizou-se abordagem cirúrgica no dia 11/12/2019, com drenagem pericárdica (Cultura negativa; albumina 2,0; LDH 1334; aspecto do líquido turvo/vermelho; hemácias 45.000 , células 5469, segmentados $91 \%$, linfócitos 5\%, monócitos 3\%, eosinófilos $1 \%$ e biópsia com pericardite aguda fibrinosa) pericardiectomia e toracotomia exploradora. Evoluiu, após o procedimento, com melhora clínica da dispneia.

Submeteu-se à radiografia de tórax de controle (do dia 14/12/2019), a qual evidenciou diminuição da área cardíaca e do derrame pleural. Foi, então, encaminhado a hospital de apoio, para dar continuidade à investigação diagnóstica.

O paciente foi admitido no serviço de clínica médica do Hospital Geral Dr. Waldemar Alcântara (HGWA) no dia 15/01/2010, com queixa principal de "dor e inchaço nas juntas", há 3 meses. Relatava que, há 3 meses da admissão no HGWA, iniciou quadro de artralgia, com edema de articulações e aumento difuso do volume abdominal. Referia que todas as suas articulações apresentavam edema, principalmente os joelhos e os tornozelos. Além disso, alegava febre diária, não aferida, sem horário preferencial e dispneia progressiva, ainda que em repouso. Por suspeita de infecção do trato urinário, encontrava-se com meropenem, iniciado no IJF dia 14/01/2020 e mantido até 05/02/2020.

Ao exame físico da admissão no HGWA, encontrava-se em bom estado geral, acianótico, anictérico, afebril ao toque, hipocorado (+1/+4), hidratado, eupneico em ar ambiente, cooperativo e algo desorientado. Notou-se presença de glossite atrófica. Na ausculta cardíaca, apresentava ritmo cardíaco regular, em dois tempos, bulhas normofonéticas, com sopro pancardíaco, sistólico, mais audível em foco aórtico, com irradiação para fúrcula. Ausculta pulmonar com murmúrio vesicular universal, diminuído em base esquerda, sem ruídos adventícios. Observado cicatriz de toracotomia à esquerda, sem sinais de flogose. Abdome globoso por adiposidade, ruídos hidroaéreos presentes, flácido, indolor à palpação superficial e profunda, sem massas ou organomegalias, timpânico à percussão. Extremidades com pulsos periféricos palpáveis, simétricos, sem edema ou cianose. Não observado deficits focais.

No dia 18/01/2020, foi aberto protocolo sepse por provável foco osteoarticular, ao apresentar sonolência, taquicardia, taquipneia, febre e edema assimétrico de joelho esquerdo (Figura 1), sendo então associada a Vancomicina.

No dia 21/01/2020, foi realizada artrocentese de joelho esquerdo, com drenagem de $190 \mathrm{ml}$ de líquido amarelo citrino (Figura 2) e, posteriormente, estudo do líquido [Hemácias: 1080; Cél. Nucleadas: 1280 (Linf: 32\%; Mono: 4\%; Neut: 64\%); Pesquisa de cristais: ausente; Cultura: negativa].

Figura 1: Edema assimétrico de joelho esquerdo

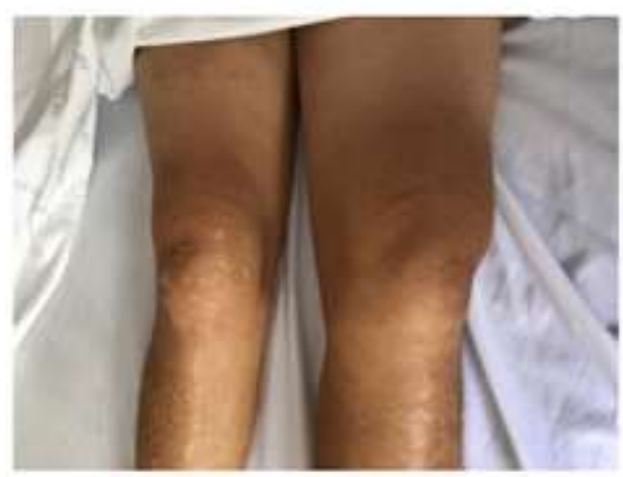

Fonte: Autores.
Figura 2: Líquido amarelo citrino proveniente da artrocentese

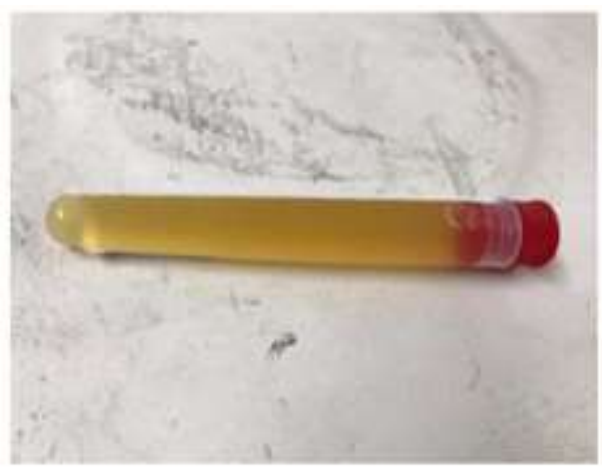

Fonte: Autores.

As Figuras 1 e 2 evidenciam o edema assimétrico de joelho esquerdo e o líquido amarelo citrino retirado de 
artrocentese, respectivamente.

No dia 23/01/2020, foi realizada troca da vancomicina por teicoplamina, devido à piora da função dom rim (Cr: 0,67 -> 1,14), sendo mantida até 05/02/2020. Por persistência dos sintomas articulares, como artralgia com edema de articulações metacarpofalangeanas, interfalangeanas proximais e distais, punhos, joelhos e tornozelos, foi iniciada prednisona (20mg/dia), apresentando melhora significativa da artralgia.

Devido à nova piora da função renal, associada à alteração na proteinúria de $24 \mathrm{~h}(11,879 \mathrm{~g})$, foi então optado pela realização de biópsia renal, onde foram evidenciadas alterações compatíveis com nefrite lúpica com sobreposição de classes III e V.

Devido às suspeitas diagnósticas de doenças autoimunes, novos exames foram realizados: anti-DNA: 1/40; FAN: 1/640 (nuclear quasi-homogêneo); anti-Sm: negativo; anticorpos anti-cardiolipina IgM e IgG não reagentes; anticoagulante lúpico não reagente; anti-histona negativo; C4 < 8; C3: 53 (consumido); anti-Ro e anti-La negativos. Além disso apresentou VHS de 130 e PCR de 5,42 (15.01). Realizou ecocardiograma transtorácico (dia 17/01/2020), o qual que evidenciou dilatação leve do átrio esquerdo, VI AE = $38 \mathrm{ml} / \mathrm{m}^{2}$; hipertrofia concêntrica leve do VE; disfunção diastólica do VE, grau I; insuficiência mitral leve; derrame pericárdico leve. Foi submetido à ultrassonografia abdominal (17/01/2020), que mostrou esteatose hepática de moderada intensidade; presença de lâmina de líquido livre anecoico na cavidade abdominal, entre alças; distensão gasosa difusa entre alças intestinais, sem evidências ecográficas de obstrução. Fez colonoscopia (28/01/2020), a qual se encontrava dentro dos padrões de normalidade. Realizou ecocardiograma transesofágico (13/02/2020), onde foram vistas valvas mitral e aórtica com espessamento dos folhetos. Além disso, viu-se imagem arredondada aderida ao folheto coronariano direito da valva aórtica, medindo $3 \mathrm{~mm}$ x 4mm, compatível com vegetação. Havia presença de insuficiência mitral leve, insuficiência tricúspide moderada, refluxo aórtico discreto e derrame pericárdico leve.

Após os exames, foi confirmado diagnóstico de Lúpus Eritematoso Sistêmico Senil. Foi iniciada terapêutica com hidroxicloroquina. O paciente apresentou melhora significativa e global das artrites, além de queda de provas de atividade inflamatória, evoluindo estável clinicamente. Recebeu alta no dia 11/02/2020, com retorno para manter acompanhamento ambulatorial.

\section{Discussão}

Os indivíduos com LES Senil (LESS) cursam com doença mais insidiosa, quando comparados aos pacientes mais jovens do Lúpus Eritematoso Sistêmico (LES) clássico (CHOI, 2015). Vale ressaltar que também são principalmente caucasianos (Riveros, 2020).

Riveros Frutos et al. (2021) realizou um estudo comparativo com 565 pacientes com LES de início tardio e 3.054 pacientes com doença de início precoce. Os achados foram consistentes com os de outras publicações, sendo evidenciado que pessoas com LES de início tardio apresentam menor envolvimento de órgãos específicos, pele, rins e articulações em comparação a pacientes de início precoce. Da mesma forma, eles apresentam mais frequentemente serosite, síndrome de Sjögren e manifestações neurológicas. A gravidade do lúpus diminui com a idade. O curso da patologia foi mais indolente no grupo de início tardio do que no grupo de início precoce, conforme refletido nos valores mais baixos de atividade da doença (SLEDAI) e no uso menos frequente de corticosteroides e drogas imunossupressoras. (Medlin, 2016)

Foi demonstrada prevalência maior de hipocomplementemia, assim como anticorpos anti-dsDNA em indivíduos com LESS. Além disso, foi evidenciado atividade da doença menos grave do que pacientes mais jovens com LES (Aljohani, 2017).

Em relação às alterações cardiovasculares demonstradas no ecotranstorácico do paciente pode estar relacionado à idade do paciente, mas também à atividade do LESS visto que estudos mostram que o risco não deve ser totalmente explicado pelo aumento da prevalência de fatores ateroscleróticos tradicionais e pode ser devido a intermediários fisiopatológicos, como 
interferons tipo I e outras citocinas inflamatórias, estresse oxidativo que resultam em lesão endotelial e dislipidemia próaterogênica. (Kostopoulou, M., 2020)

No relato descrito, a hipótese inicial diagnóstica para paciente em questão não foi LES, devido a diversos fatores, como a baixa prevalência do LES Senil, que é relativamente raro, assim como pelo fato de o paciente ser do sexo masculino (1:4).

O diagnóstico do LES é dado com os critérios da Liga Europeia contra o Reumatismo (EULAR) e do Colégio Americano de Reumatologia (ACR) de 2019. O critério de entrada é a presença de FAN >= 1:80. Além disso, são adotados critérios clínico-laboratoriais. Vale ressaltar que em casos com mais de um critério por sistema, é somado apenas o sintoma de maior pontuação. O diagnóstico é dado quando a pontuação dos critérios for maior ou igual a dez pontos (Aringer, 2019).

O paciente em questão pontuou por artrite (06 pontos), febre (02 pontos), serosite (05 pontos), proteinúria de $24 \mathrm{~h}$ de 11,8g, biópsia renal classe III e IV (08 pontos), anti-DNA 1:40, C3 e C4 consumidos (06 pontos), totalizando 27 pontos.vDe acordo com o estudo de Medhat et al. (2020), o principal desafio do tratamento de pacientes com LESS decorre da alta prevalência de comorbidades, como osteoporose, hipotireoidismo, diabetes mellitus e hipertensão. Além disso, há maior prevalência de multimorbidade (comorbidades $\geq 2$ ) nesses casos (Ilar, 2019).

No caso do paciente, ele tinha como comorbidade relevante apenas a hipertensão. Observou-se boa evolução com a terapêutica inicial com corticoide e a manutenção com a hidroxicloroquina (Gurevitz, 2013). A melhora imediata com prednisolona e hidroxicloroquina também foi evidenciada em outros estudos.

\section{Conclusão}

O diagnóstico precoce do Lúpus Senil é desafiador, dada à pluralidade de sintomas, assim como a baixa frequência da doença na faixa etária tardia. Apesar de raro, o LESS deve sempre ser lembrado como diagnóstico diferencial, que requer como primeiro passo a investigação mediante o FAN.

Estudos demonstram que a atividade da doença no LESS cursa com complicações menos severas, todavia não são irrelevantes, uma vez que os pacientes senis costumam ter maior número de comorbidades.

Por isso, sugere-se, que mais pesquisas sejam realizadas continuamente na área de diagnósticos precoces, contribuindo para que tais pacientes sejam adequadamente tratados, o que reduz o tempo de internamento hospitalar, diminuindo custos e propiciando maior segurança ao paciente.;

\section{Referências}

Arnaud, L., Mathian, A., Boddaert, J., \& Amoura, Z. (2012). Late-onset systemic lupus erythematosus. Drugs \&amp; aging, 29 (3), 181-189. doi: 10.2165 / 11598550-000000000-00000

Frutos, A. R.; Holgado, S.; Bergé, A. S.; Casas, I.; Olivé, Alejandro.; Longo, F. J. L.; Alén, J. C.; Galindo, M.; Nebro, A. F.; Reigosa, J. M. P.; Figueroa, I. R.; \& Relesse, G. (2021). Late-onset versus early-onset systemic lupus: characteristics and outcome in a national multicentre register (RELESSER). Rheumatology, 60 (4), 1793-1803. doi: 10.1093 / reumatology / keaa477.

Lalani, S., Pope, J., Leon, F., Peschken, C., \& Canios, M. (2010). Clinical features and prognosis of late-onset systemic lupus erythematosus: results from the 1000 faces of lupus study. The Journal of rheumatology, 37 (1), 38-44. doi: 10.3899 / jrheum.080957.

Choi, J. H., Park D. J., Kang J. H., Yim, Y. R., Lee, K.E., Lee, J. W., Wen, L., Kim, T. J., Park, Y. W., Lee, J. K.; \& Lee, S. S. (2015). Comparison of clinical and serological differences among juvenile-, adult-, and late-onset systemic lupus erythematosus in Korean patients. Lupus, 24 (12), 1342-1349. doi: 10.1177 / 0961203315591024.

Medhat, B. M., Behiry, M.E., Sobhy, N., Yomna, F., Marzouk, H., Mostafa, N., Khalifa, I., Elkhalifa, M., Eissa, B. M., \& Hassan, E. H. E. (2019). Late-onset systemic lupus erythematosus:characteristics and outcome in comparison to juvenile-and adult-onset patients-a multicenter retrospective cohort. Clinical rheumatology, 39, (2), 435-442. doi: 10.1007 / s10067-019-04776-y.

Aringer, M., Costenbader, K., Daikh, D., Brinnks, R., Mosca, M., Goldman, R. R., Smolen, J. S., Wofsy, D., Boumpas, D. T., Kamen, D.L., Jayne, D., Carvera, R., Chalumeau, N.C., Diamond, B., Gladman, D. D., Hahn, B., Hiepe, F., \& Jacobsen, S. (2019). 2019 European League Against Rheumatism/American College of Rheumatology classification criteria for systemic lupus erythematosus. Arthritis \&amp; rheumatology, 71, (9), 1400-1412. doi: 10.1002 / art.40930. 
Lucic, A. T., Petrovic, R., Perovic, M.R., Milovanovic, D., Milovanovic, J., Zivanoviz, S., Pentovic, S., \& Veselinovic, M. (2013). Late-onset systemic lupus erythematosus: clinical features, course, and prognosis. Clinical rheumatology, 32, (7), 1053-1058. doi: 10.1007 / s10067-013-2238-y.

Cildag, S., Kara, Y., Cakir, E., Cildag, M. B., \& Senturk, T. (2019). Comparison of clinical and laboratory findings in patients with systemic lupus erythematosus with regard to age at onset. The Eurasian journal of medicine, 51, (1), 17. doi: 10.5152/ eurasianjmed.2018.18022.

Sohn, I. W., Joo, Y. B., Won, S., \& Bae, S. C. (2018). Late-onset systemic lupus erythematosus: Is it "mild lupus"?. Lupus, 27, ( 2), 235-242.doi: 10.1177 / 0961203317716789.

Medeiros, M. M. C., Bezerra, M. M. C., Braga, F. N. H. F., Feijão, M. R. M. J., Gois, A. C. R., Rebouças, V. C. R., Carvalho, T. M. A. Z., Carvalho, L. N. S., \& Ribeiro, A. T. M. (2016). Clinical and immunological aspects and outcome of a Brazilian cohort of 414 patients with systemic lupus erythematosus (SLE): comparison between childhood-onset, adult- onset, and late-onset SLE. Lupus, 25, (4), 355-363. doi: 10.1177 / 0961203315606983.

Aljohani, R., Gladman, D. D., Su, J., \& Urowitz, M. B. (2017). Evolution of late and early onset systemic disease lupus erythematosus. Lupus. 26 (11): 11901196. doi: 10.1177 / 0961203317696593.

Medlin, J. L. M. D., Hansen, M. D. M. S., Fitz, M. D. S. R., \& Bartels, M. D. C. M. (2016). Uma revisão sistemática e meta-análise das manifestações cutâneas no lúpus eritematoso sistêmichanco de início tardio versus precoce. Seminários em Artrite e Reumatismo, 45 (6), 691-697. https://doi.org/10.1016/j.semarthrit.2016.01.004

Chen, Y. M., Lin C. H., Chen, H.H., Chang, S. H., Hung, W. T., Lai, K. L., Lan, J. L., Chen, D. Y., \& Lan, T. H. (2014). Onset age affects mortality and renal outcome of female systemic lupus erythematosus patients: a nationwide population-based study in Taiwan. Rheumatology, 53 (1), 180-185. https://doi.org/10.1093/rheumatology/ket330

Lazaro, D. (2007). Lúpus eritematoso sistêmico de início na terceira idade. Drogas e envelhecimento, 24 (9): 701-715. doi: 10.2165 / 00002512-20072409000001

Golmar, A. C., Moriano, C., Garijo, M., \& Gonzalez, I. (2018). Clinical-epidemiological features of patients with a late-onset lupus in a tertiary care hospital. Annals of the Rheumatic Diseases, 77 (6), 1431. doi: 10.1136/annrheumdis-2018-eular.7538

Gurevitz S. L., Snyder J. A., Wessel E. K., Frey J., \& Williamson B. A. (2013). Lúpus eritematoso sistêmico: uma revisão da doença e opções de tratamento. Consult Pharm, 28 (2): 110-21. doi: 10.4140 / TCP.n.2013.110.

Kaul, A., Gordon, C., Crow, M. K., Touma, Z., Urowitz., Vollenhoven, R. V., Irastorza, G. R., \& Hughes, G. (2016). Lúpus Eritematoso sistêmico. Nat Ver Dis Primers. 2: 16039. Doi: 10.1038 / nrdp.2016.39

Estel, G. J. P., Gil, M. F. U., \& Alarcon, G. S. (2017). Epidemiologia do lúpus eritematoso sistêmico. Expert Rev Clin Immunol. 13 (8): 799-814. doi: 10.1080 / 1744666X.2017.1327352

Kostopoulou, M., Nikolopoulos, D., Parodis, I., \& Bertsias, G. (2020). Doença cardiovascular no lúpus eritematoso sistêmico: dados recentes sobre epidemiologia, fatores de risco e prevenção. Curr Vasc Pharmacol. 18 (6): 549-565. doi: 10.2174 / 1570161118666191227101636.

Montjoye, S., Boland, B., Raemdonck, J. V., \& Houssiau, F. A. (2020). Lúpus eritematoso sistêmico de início muito tardio como causa incomum de deficiências funcionais e cognitivas reversíveis em um paciente octogenário. Eur J Case Rep Intern Med, 7 (8): 001570. doi: 10.12890 / 2020_001570

Pereira A. S. et al. (2018). Metodologia da pesquisa científica. [free e-book]. Ed. UAB/NTE/UFSM. 